\title{
Modifying the Hall Plot for Analysis of Immiscible Gas Injection Wells II: IOR
}

\author{
Oluwole A. Talabi and Zhen-Xuan Yew
}

\begin{abstract}
The Hall plot is a graph of an injection well's cumulative pressure against its cumulative injected volume, ideally presenting as straight line. Changes in the gradient of this line can indicate a change in injectivity behavior. Developed for single-phase, steady-state, radial flow of liquids, it has proven useful in the surveillance of water injection wells. However, published studies into the validity of its equations and assumptions for gas injection scenarios are limited. Utilizing the Hall plot to analyze immiscible gas injection into an oil phase for improved oil recovery poses challenges due to the near-wellbore relative permeability of gas to the oil phase. This paper presents one useful and practical approach to achieve this correction.

To account for the varying near-wellbore relative permeability of gas in the oil phase, a function relating the relative permeability of gas in the oil phase to the cumulative gas injection is developed and included in previously developed equations that account for compressibility and viscosity variations. These updated equations give a modified Hall plot that accounts for the non-linearity due to relative permeability effects and allows the Hall plot to be used qualitatively by engineers to identify changes in the injectivity of gas wells.
\end{abstract}

Index Terms - Gas, injection, immiscible, EOR.

\section{INTRODUCTION}

The interpretation of rate and pressure data associated with gas injection schemes for IOR/EOR is key to the efficient management and control of the injection wells. One such tool utilized by engineers is the Hall plot which has found application as a surveillance tool to indicate when a change in injectivity of the well has occurred, and, if sufficient data is available, estimate the magnitude of this change.

The Hall plot [1] is a plot of cumulative injection pressure against the cumulative volume injected used to analyze well injectivity over a period of time. Changes in the gradient of the plot indicate potential changes in well conditions which should be investigated. Due to its simplicity and the usual availability of required data, the Hall plot is a common surveillance and monitoring tool in injection projects. It was originally developed from Darcy's radial flow equation and assumes the injected fluid is incompressible, single-phase, reservoir pressure is constant, flow is steady-state and that the bottomhole pressure and wellhead pressure can be related by correction of hydrostatic head and friction losses in the wellbore. Since then, it has also been modified and applied in several ways, extending its application.

Buell et al [2] modified it for polymer injection in EOR. Ojukwu and van den Hoek [3] modified it to a semi-steady

Manuscript received February 18, 2018; revised March 30, 2018.

Oluwole A. Talabi and Zhen-Xuan Yew are with Schlumberger Oilfield Services, Kuala Lumpur, Malaysia (e-mail: otalabi@slb.com, zyew@slb.com). state version for use in quantifying damage skin. Silin et al, [4] developed slope analysis to estimate and use the varying reservoir pressure term. Izgec and Kabir [5] completely reformulated the Hall plot in a way that accounts for variable radial distance of the injection bank and pressure at the water/oil interface. They also included a companion derivative curve to obtain clearer signatures of plugging or fracturing. This combination of curves has been called a modified or advanced Hall plot.

However, only limited literature is available covering scenarios with gas being injected into the liquid phase. While Laochamroonvorapongse et al [6] applied the method of Izgec and Kabir [5] to both miscible and immiscible WAG floods, they did not discuss the validity of their assumptions for the gas injection cycles. Izgec [7] demonstrated an application of the advanced Hall plot for gas injection wells but he notes, without discussion, that its assumptions are only valid for gas wells when the pressure is above 5000 psia where most hydrocarbon gases behave like liquids.

There are additional complications found when applying the Hall plot to gas injection, namely: i) the significant pressure dependence of gas fluid properties and ii) the variation of relative permeability with saturation. Talabi, [8] discusses the first complexity by examining the applicability of, and modifications to, the Hall plot for immiscible gas injection into another gas phase. The conclusions align with Izgec's [7] comments, showing that the Hall plot may be used above a pressure of 4000 psia or, more specifically with regard to gas composition, above a reduced pressure of 4 . Below such pressures, a different expression is to be used.

Utilizing the Hall plot for immiscible gas injection into an oil phase such as in an EOR scheme brings us to the second complexity: changing near-wellbore saturation, and thus, relative permeability of gas, in the oil phase. A correction must be applied in order to account for this. This article presents one such proposed approach this correction.

\section{Overview OF Original Hall Plot}

The Hall plot starts at the radial flow equation and assumes single-phase, steady-state, flow of a well centered in a circular homogenous reservoir. Fluids in the well and reservoir are homogeneous and incompressible. The reservoir is also assumed to be vertically confined and uniform in terms of permeability and thickness. Writing the radial flow equation and integrating both sides, we get:

$$
\sum[\Delta P \cdot \Delta t]=\frac{\mu\left(\ln \frac{r_{e}}{r_{w}}+S\right)}{0.00708 k h} * Q_{w}
$$

Which can be written as 


$$
\sum \Delta P \cdot \Delta t=\frac{Q_{w}}{C_{w}}
$$

This equation can be plotted as a simple straight line of slope $1 / C_{w}$.

For incompressible liquids, the bottomhole pressure and wellhead pressure can be related by correction of hydrostatic head and friction losses in the wellbore and that reservoir pressure may be approximated as constant. This allows a simple implementation of the Hall plot as reported in his original paper whereby cumulative wellhead pressure is often plotted against cumulative water injection. By plotting this, Hall observed that if an injection well was stimulated, the slope of the plot decreased and if the well is damaged, the slope increased.

\section{Methodology}

\section{A. Formulating the Correction for Immiscible Gas Injection into an Oil Phase}

Talabi [8], discussed the complexity of application of Hall plot for immiscible gas injection into another gas phase by modifying it to account for viscosity and compressibility variation with pressure assuming constant relative permeability which would be the case for single phase injection. The low-pressure approximation was found to be adequate for all pressures while the high pressure (original Hall plot) was only adequate for pressures over 4000psia. They are given in equations 3 , and 4 respectively.

$$
\begin{gathered}
\sum\left[\frac{\left(P_{w i}{ }^{2}-P_{R}{ }^{2}\right)}{\bar{\mu} \bar{z}} \cdot \Delta t\right]=\frac{Q_{g}}{C_{1}} \\
\sum \Delta P \cdot \Delta t=\frac{Q_{g}}{C_{2}}
\end{gathered}
$$

Both of which can be plotted as straight lines of slope $1 / C_{1}$, $1 / C_{2}$ in the manner of Hall.

The effective permeability, $k$ however, is composed of the product of the absolute permeability, $K_{a b}$ and relative permeability of gas in oil, $k_{r g}$. This relative permeability cannot be assumed constant when injecting a gas into a liquid phase, such as in EOR. Therefore, the equations can be modified by assuming that at every steady state condition, there is some representative, average, near-wellbore relative permeability of gas in the oil phase that depends only the total amount of gas injected. This is reasonable, since the relative permeability depends on gas saturation which in turn increases with the total amount of gas injected as near wellbore pore spaces that previously contained oil are filled with gas. Writing this average relative permeability as $\overline{k_{r g}}$ and rederiving the modified Hall expressions without assuming it to be constant, then the previously presented equations may be further changed.

The low-pressure formulation becomes:

$$
\sum\left[\overline{k_{r g}} \cdot \frac{\left(P_{w i}{ }^{2}-P_{R}{ }^{2}\right)}{\bar{\mu} \bar{z}} \cdot \Delta t\right]=\frac{Q_{g}}{C_{3}}
$$

The high pressure (original Hall plot) expression becomes:

$$
\sum \overline{k_{r g}} \cdot \Delta P \cdot \Delta t=\frac{Q_{g}}{C_{4}}
$$

These can be plotted as straight lines of slope $1 / C_{3}$ and $1 / C_{4}$ respectively, in the manner of Hall, provided that the correction $\overline{k_{r g}}$ can be evaluated using the typical Hall plot data.

\section{B. Defining the Correction Factor as a Function of Cumulative Gas Injection}

We can write the form of a general relationship between relative permeability of gas, $k_{r g}$ with gas saturation, $S_{g}$, the maximum gas relative permeability, $k_{r g, \max }$ and the Corey-type exponent, $n_{g}$.

$$
k_{r g} \propto k_{r g, \max }\left(\frac{s_{g}}{s_{g, \max }}\right)^{n_{g}}
$$

It can also be shown from material balance and Buckley-Leverett analysis [9] that the average saturation behind the flood front is directly proportional to the square-root of cumulative gas injected through the well:

$$
\overline{\overline{S_{g}}} \propto \sqrt{Q_{g}}
$$

Therefore, if we assume that the average gas saturation behind the front is related to average relative permeability we defined earlier $\overline{k_{r g}}$, we can then write a relationship between the square-root of cumulative gas injected at any point in time and the average gas relative permeability in the gas-oil system as:

$$
\overline{k_{r g}}=k_{r g, \max }\left(\frac{\sqrt{Q_{g}}}{Q_{g, \max }}\right)^{m_{g}}
$$

where $m_{g}$ is an exponent to be determined for each case and $Q_{g, \max }$ is some arbitrarily high volume of gas injected. For simplicity, it can be set equal to one pore volume of the reservoir. As will be presented in the following sections, the actual value used here has no significant impact on the linearity of the resulting plot. It need only be positive. Therefore, the correction $\overline{k_{r g}}$ may now be used when injecting gas into oil reservoirs to account for the effect of changing relative permeability when we analyse the production data.

\section{Testing the Approach}

The proposed correction is tested using a commercial reservoir simulator. A simple model with one injector and one producer is used. The Dykstra-Parsons coefficient [10] is used as a simple indicator of the degree of heterogeneity. For the first group of experiments, we used one set of relative permeability curves (Rock type A) along with a homogenous model to investigate the effectiveness of the new formulation in straightening the Hall plot when gas is injected into an oil phase. Following this, we tested the impact of increasing heterogeneity on the effectiveness of the method in order to establish its robustness. The overall average permeability of all the models are kept the same. Third, we tested the method using different sets of relative permeability curves, to establish its applicability to different rock types. The curves used are labelled Rock Type A, B, and Berea. A and B are synthetic rock curves while The Berea rock type are the 
laboratory reported curves for flow perpendicular to the bedding plane of the Berea sandstone reservoir as reported by Corey and Rathjens [11]. In all the experiments performed, the model was saturated with oil and throughout the injection period, average reservoir pressure remained 850 - 3500 psia. This was achieved by adjusting oil production and gas injection rates. The data generated from the experiments were used to test the applicability of the relative permeability correction.

\section{Results}

The simulation results of bottomhole pressure, average reservoir pressure and cumulative gas injection were extracted and plotted using the uncorrected, original Hall plot in Fig. 1. Since gas was being injected into the oil zone, it was expected to see that the plot is not a straight line as shown. The same data was plotted using the low-pressure approximation (Fig. 2). A straight line cannot be seen on the Hall plot because of changing relative permeability.

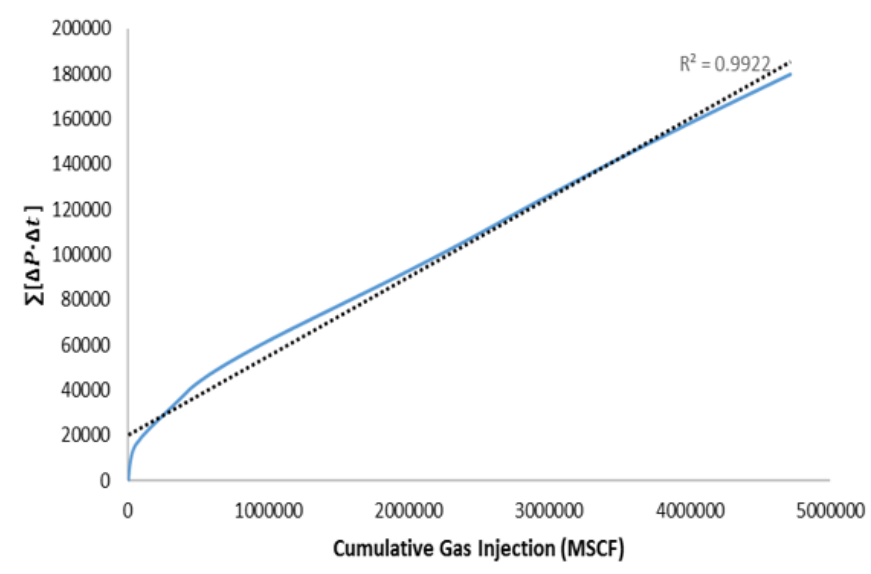

Fig. 1. Original hall plot for rock A (uncorrected).

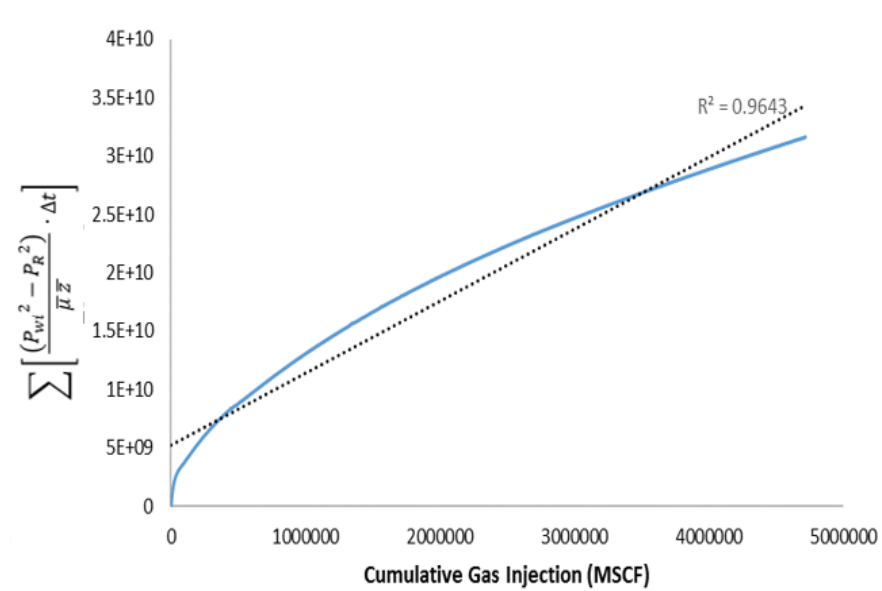

Fig. 2. Low pressure approximation for rock A (uncorrected).

Using the same data, we regenerated the original Hall plot and low pressure formulation including the gas relative permeability correction. We observed some improvement of the original Hall plot (Fig. 3) however, the corrected low pressure formulation (Fig. 4) is most improved and now a straight line since both pressure and relative permeability are accounted for.

\section{A. Impact of Heterogeneity on the Correction Factor.}

In deriving the new equations, we maintained the assumption of homogenous reservoir properties and perfect radial displacement. In order to test the sensitivity of the correction to deviations from these assumptions which are generally not valid in real cases, we introduced increasing levels of heterogeneity (estimated using the Dykstra-Parsons Coefficient) into the test models. The results from each of these cases were plotted using the low-pressure formulation with and without correction in Fig. 5 to Fig. 8. Results show that even with high heterogeneity, the linearity of the plot was always improved.

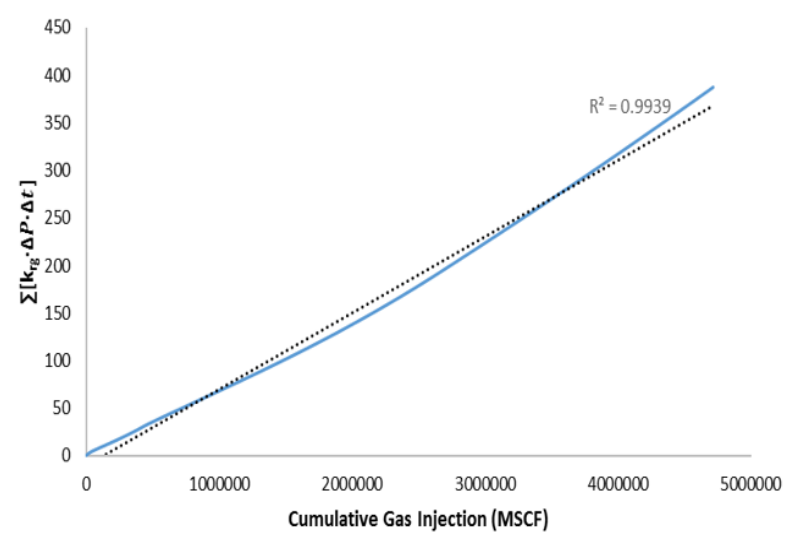

Fig. 3. Original hall plot with Krg correction for rock A.

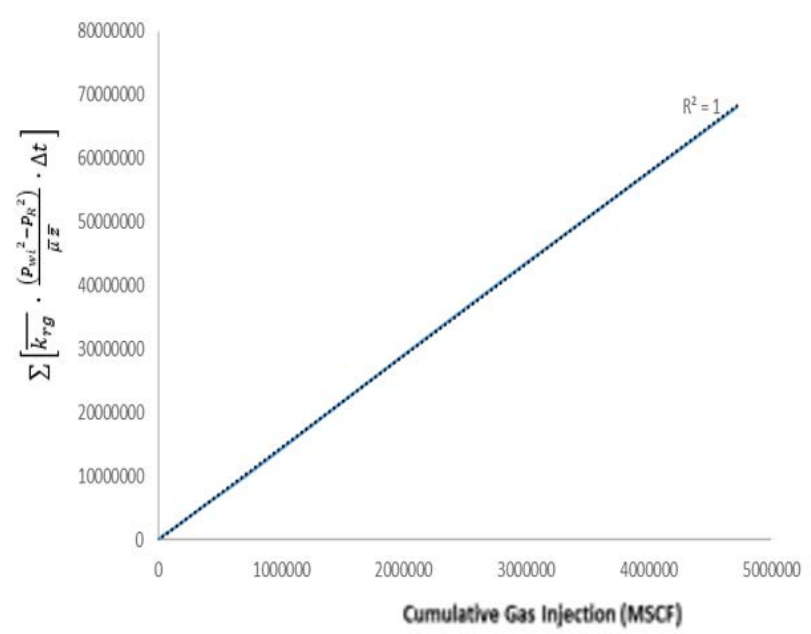

Fig. 4. Low pressure approximation with $\mathrm{K}_{\mathrm{rg}}$ correction for rock $\mathrm{A}$.

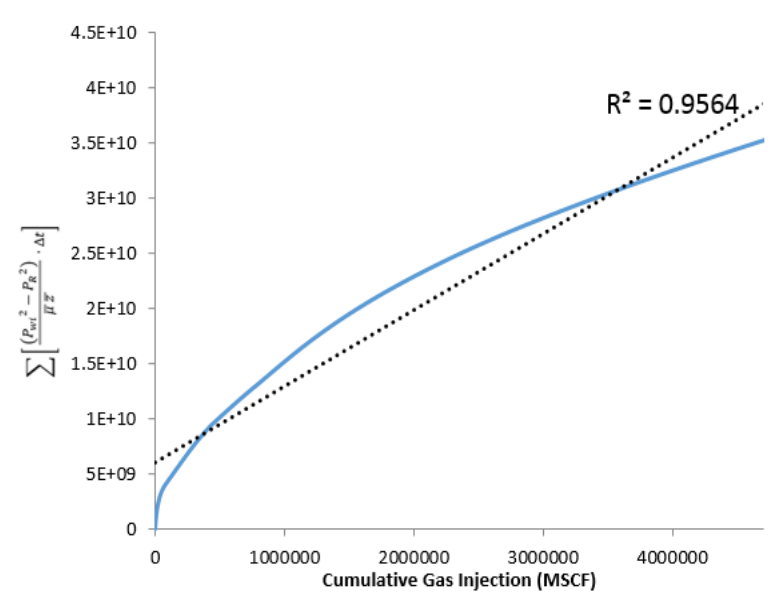

Fig. 5. Uncorrected low pressure formulation results in DP-0.25 model. 


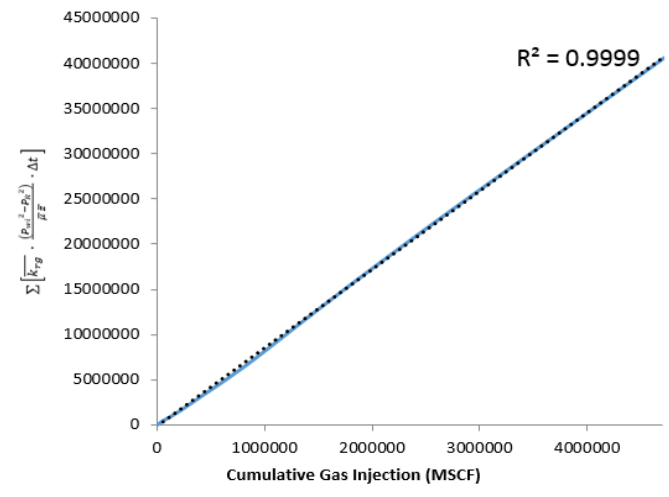

Fig. 6. Low pressure formulation results with correction in DP-0.25 model.

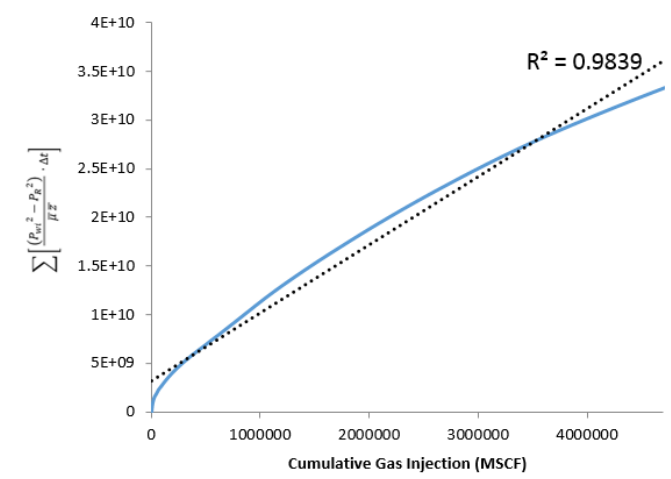

Fig. 7. Uncorrected low pressure formulation results in DP-0.91 model.

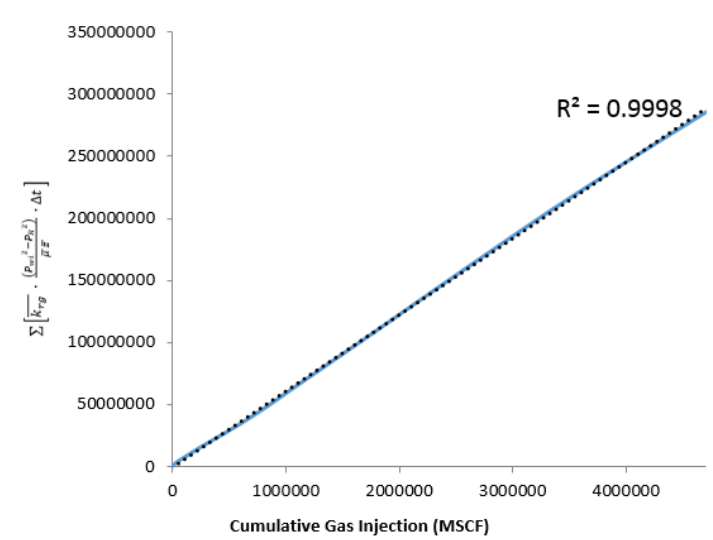

Fig. 8. Low pressure formulation results with correction in DP-0.91 model.

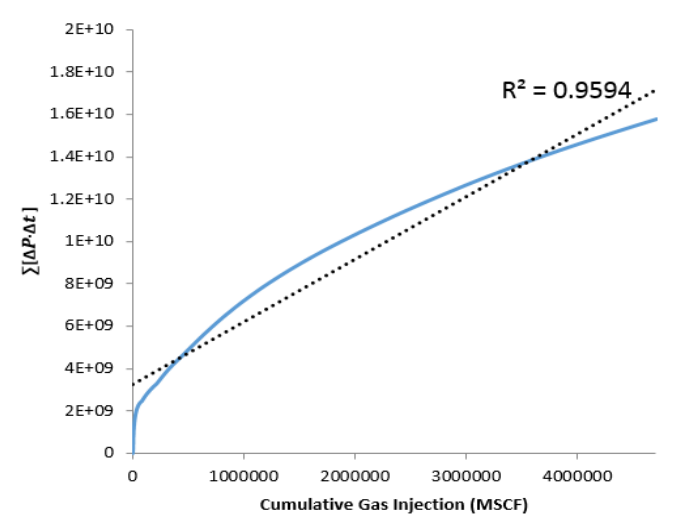

Fig. 9. Uncorrected low pressure formulation results for Rock B.

\section{A. Applicability to Different Reservoir Rock Types}

As the previous experiments were conducted using Rock A, the next experiments used Rocks $B$ and Berea to test the correction. With different sets of relative permeability curves representing the different rock types, we observed similar results as in previous experiments: the corrected original Hall plot results shows improvement but does not straighten the line completely. The low-pressure formulation with the gas relative permeability correction does as shown in Fig. 9 to 12.

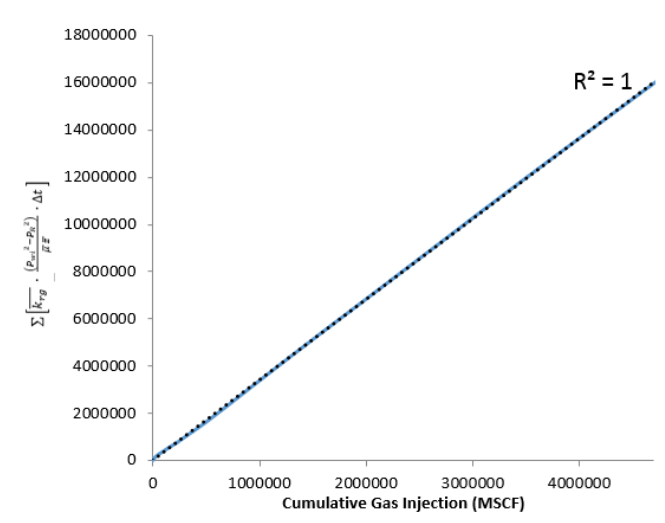

Fig. 10. Low pressure formulation results with Krg correction factor for Rock B.

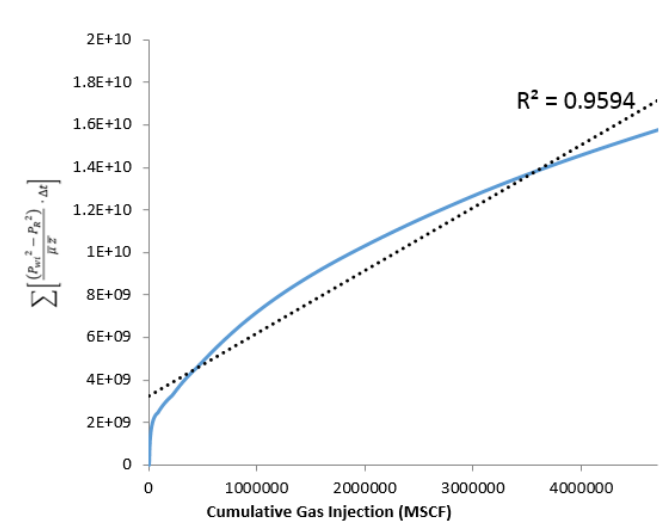

Fig. 11. Uncorrected low pressure formulation results for Rock Berea.

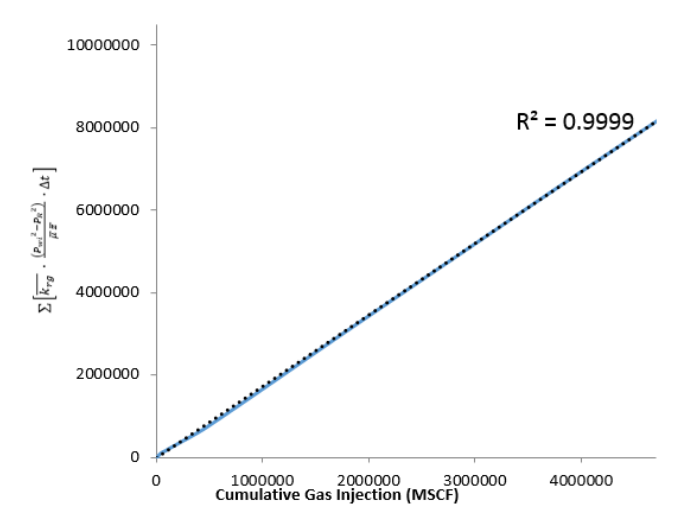

Fig. 12. Low pressure formulation results with Krg correction factor for Rock Berea.

\section{CONCLUSION}

From the results presented above, the following conclusions may be drawn:

1) Hall plot analysis of immiscible gas injection into an oil phase without accounting for varying compressibility, viscosity and near-wellbore relative permeability of gas to the oil phase can be misleading due to deviations from straight line behaviour.

2) Previous work has demonstrated modifications to account for the pressure dependence of compressibility and viscosity and an additional correction to those previously modified Hall plot equations to account for changing near-wellbore relative permeability can be 
obtained as a function of cumulative gas injection.

3) The proposed gas relative permeability correction helps to straighten out the plot, and while it doesn't perfectly linearize the high-pressure simplification (original Hall plot), it significantly linearizes the low pressure formulations, allowing changes in injectivity performance to be effectively identified.

4) The results further showed that the correction remains useful even with increasing heterogeneity and over a range of rock types.

Overall, this work indicates that a simple correction to the previously modified versions of the Hall plot can be used qualitatively by engineers to identify injectivity problems during injection of gas into hydrocarbon reservoirs - in particular, immiscible gas injection into a gas cap and immiscible gas injection into an oil reservoir - as long the appropriate equation is selected for the operating pressure range and the effects of compressibility, viscosity and relative permeability are accounted for.

\section{REFERENCES}

[1] H. N. Hall, "How to analyze waterflood injection well performance," World Oil, pp. 128-130, Oct. 1963.

[2] R. S. Buell, H. Kazemi, and F. H. Poettmann, "Analyzing injectivity of polymer solutions with the hall plot," SPE Journal of Reservoir Engineering, vol. 5, issue 1, Feb. 1990.

[3] K. I. Ojukwu and P. J. Van den Hoek, "A new way to diagnose injectivity decline during fractured water injection by modifying conventional hall analysis,” presented at the SPE/DOE Symposium on Improved Oil Recovery, Oklahoma, April 17-21, 2004.

[4] D. B. Silin, R. Holtzman, T. W. Patzek, J. L. Brink, and M. L. Minner, "Waterflood surveillance and control: Incorporating hall plot and Slope analysis," in Proc. SPE Annual Technical Conference and Exhibition, ATCE, Apr. 2005, pp. 1191-1205.
[5] B. Izgec and C. S. Kabir, "Real-time performance analysis of water-injection wells,” SPE Reservoir Evaluation \& Engineering, vol. 12 issue 1, pp. 116-123, Feb. 2009.

[6] R. Laochamroonvorapongse, C. S. Kabir, and L. W. Lake, "Performance assessment of miscible and immiscible water-alternating gas floods with simple tools," Journal of Petroleum Science and Engineering, vol. 122, pp. 18-30, Sep. 2014.

[7] B. Izgec, "Integrated injection modeling," in Proc. the SPE Annual Technical Conference and Exhibition, Houston, Texas, USA, September 28-30, 2015.

[8] O. A. Talabi, "Modifying the hall plot for analysis of immiscible gas injection wells," in Proc. the Offshore Technology Conference Asia, Kuala Lumpur, Malaysia, March 22-25, 2016.

[9] S. E. Buckley and M. C. Leverett, "Mechanism of fluid displacement in sands,” Transactions of the AIME, vol. 146, issue 1, Dec. 1942.

[10] H. Dykstra and R. L. Parsons, "The prediction of oil recovery by waterflooding," in Secondary Recovery of Oil in the United States, 2nd Ed., Washington, DC: American Petroleum Institute, pp. 160-174 1950.

[11] A. Corey and C. Rathjens, "Effect of stratification on relative permeability,” Journal of Petroleum Technology, vol. 8, issue 12, pp. 69-71, Dec. 1956.

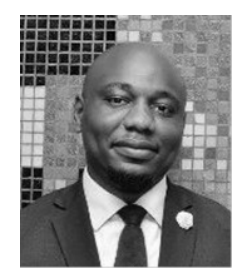

Oluwole A. Talabi got B.Sc. in chemical engineering from Obafemi Awolowo University, Ile-Ife (2008) and M.Sc. and DIC from Imperial College, London (2011). He is a senior reservoir engineer in Schlumberger Oilfield Services, Kuala Lumpur, Malaysia.

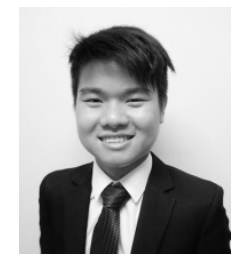

Zhen-Xuan Yew got B.Sc. in chemical engineering from Universiti Malaya, Malaysia (2015). He is a reservoir engineer in Schlumberger Oilfield Services, Kuala Lumpur, Malaysia. 